\title{
Preservation and change of hue, brightness, and form in apparent motion
}

\author{
DAVID NAVON \\ University of Haifa, Haifa, Israel
}

\begin{abstract}
Subjects were presented with several ambiguous apparent motion situations, in which the elements of each single array differed in hue, brightness, or form. One of the interpretations of each situation preserved the hue (or brightness or form) of each element across arrays. The others did not. It was found that the type of motion perceived is not biased at all toward preserving brightness and form and that hue tends to be preserved in one situation but not in others. The findings are interpreted to support the notion that processing of motion, color, and form is done by parallel processes that may have different processing rates and that may utilize each other's final or intermediary output.
\end{abstract}

It is well documented that apparent motion is perceived despite considerable disparities between stimuli (e.g., Burt \& Sperling, 1981; Kolers \& Pomerantz, 1971; Navon, 1976; Neuhaus, 1930; Rattleff, 1956). However, it has been suggested (e.g., by Higginson, 1926; Neff, 1936; Orlansky, 1940) that although disparity between stimuli does not rule out apparent motion, it may nevertheless make it less compelling or likely. To study this issue, Navon (1976) presented subjects with several situations of ambiguous apparent motion. One interpretation of each situation preserved the figural identity of the elements in the visual field across the alternated displays. The other interpretation(s) did not. Navon found that preservation of shape failed to bias perception of motion except when the figural analysis involved was relatively simple or when shape disparity was accompanied by a considerable size disparity.

The same rationale was employed here to study the effect of color disparity: If color identity has any effect at all on the formation of motion impression, then given the choice of perceiving either a motion that preserves the color or an otherwise equally likely motion that does not, the visual system should favor the first one.

To compare the effect of color with the effect of shape, I also replicated part of the experiment reported by Navon (1976).

\section{EXPERIMENT 1}

\section{Method}

Stimuli. Each different situation of apparent motion may be called an episode. An episode is a sequence of repetitive cycles of two different arrays. Each array was presented for $180 \mathrm{msec}$ with a 30 -msec time interval between any two consecutive arrays, as well as between any two consecutive cycles. An array consisted of two or six elements of one of the following types: (1) six line segments with the same centers but varying in orientation, (2) six gray disks varying in brightness, (3) six colored disks varying in hue (but intensity and chroma were not controlled). The line segments were drawn with a Leroy standard reservior pen (No. 00) on white cards. Actual orientations used were $0,30,60,90,120$, and $150 \mathrm{deg}$. The gray disks were cut out of Letratone sheets and applied to white cards. The sheets were composed of dot patterns with density of $25.5 \mathrm{lines} / \mathrm{cm}$. The percentages of black area actually used ranged from $20 \%$ to $70 \%$ in steps of $10 \%$ (corresponding Letratone sheet numbers were LT51, LT52, LT53, LT54, LT55, and LT56). The colored disks were cut out of sheets of colored paper and applied to white cards. The colors used were red (approximately 6.5R $4.0 / 13$ by Munsell notation), yellow (approximately $4.0 \mathrm{Y}$ 9.5/7), green (approximately 6.0G 6.0/5), blue (approximately 7.5PB 3.2/16), purple (approximately 6.0P 6.0/12), and orange (approximately 5 YR 8.0/10). The arrays in one cycle of each of the five episodes used in this experiment and possible interpretations for the episodes are illustrated in Figure 1.

In the "seesaw" episodes, there were only two elements in each array. The element sets used were: (1) vertical and horizontal line segments, (2) gray disks with black contents of $70 \%$ and $20 \%$, (3) gray disks with black contents of $60 \%$ and $30 \%$,

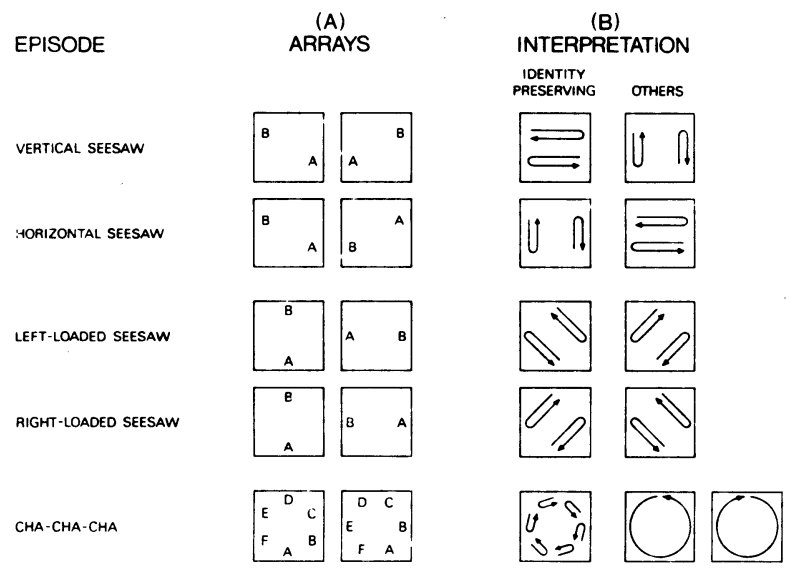

Figure 1. The arrays in one cycle of each of the five episodes and most likely interpretations of the episodes. The arrays of the different episodes are presented in $\mathbf{A}$ in order of their appearance. Different letters stand for different colors or line segments. Several possible interpretations of the episodes are presented in B. A perceived motion is represented by an arrow tracing its direction and approximate location. Size relationships are distorted due to space limitations. The fixation point is omitted from the figure. 
(4) gray disks with black contents of $50 \%$ and $40 \%$, and (5) red and green disks.

In the "cha-cha-cha" episode, all six elements of an element type appeared in each array. The assignment of elements to positions was done to avoid as much as possible any circular order. The locations of the elements of the second array in this episode were halfway between two adjacent locations of the elements of the first array.

Subjects. Subjects were 20 nonpaid undergraduates at the University of Haifa who participated in the experiment as part of their requirement in the Department of Psychology. All subjects had normal or corrected vision.

Design. The five episodes were paired with the five element sets according to a 5 by 5 Graeco-Latin square. Each subject was assigned to one sequence of the Graeco-Latin square.

Apparatus and setting. Stimuli were presented via a threefield Harvard tachistoscope (Model T-3B-1). Viewing distance was $76 \mathrm{~cm}$. The length of a line segment, as well as the diameter of a disk, subtended 0 deg $23 \mathrm{~min}$ of visual angle. The distance between element centers in each array of the "seesaw" episodes, as well as the distance between two opposite disk centers in each array of the "cha-cha-cha" episode, subtended about $3 \mathrm{deg}$ of visual angle. The luminance of the fields was set at about $12.0 \mathrm{~cd} / \mathrm{m}^{2}$.

Procedure. At the beginning of the session, the experimenter read general instructions and asked the subject to attend to his immediate impression rather than try to describe the episode analytically. The subject also was asked to fixate at a center dot that was continually present when watching the stimuli. Each trial was preceded by a verbal "ready" signal. The episode was then presented for about $5 \mathrm{sec}$. After the termination of the presentation, the subject was asked to describe verbally his interpretation of the episode, and in case of difficulties in verbal description, he could use some rods and buttons that were placed on the table to physically demonstrate his experience.

\section{Results}

The "seesaw" episodes. Only one subject reported switching of motion interpretations in the "seesaw" episodes, so it was decided to include in the data only the first interpretation of every subject.

First, let us inspect data about the "horizontal seesaw" and "vertical seesaw" episodes. When data were pooled across element sets, no association between the observed interpretation of an episode and the one predicted by identity preservation was found [Cochran's $\mathrm{Q}(1)=2.0, \mathrm{p}>.10]$. Identity was preserved in both episodes for only 1 subject of 20 . For four more subjects, identity was preserved in neither of the two episodes, but by and large (in 15 cases; two-tailed binomial probability $=.04$ ), a subject interpreted both episodes in the same way, which means that he was not affected by the identity of the elements.

Data from the "loaded seesaw" episodes also did not indicate any effect of element identity. No association between the observed interpretation of an episode and the one predicted by an identity preservation was found [Cochran's $Q(1)=0, p=1.0]$. Again, only one subject reported identity-preserving motion in both episodes. For two more subjects identity was not preserved in both episodes, and 14 subjects reported identical interpretations in both episodes (the data of three more subjects were unclear in this respect; after eliminating them, binomial probability $=.01$ ).
Table 1

Frequencies of Interpretations That Do (P) and Do Not (NP) Preserve Element Identity in the Four "Seesaw" Episodes, Tabulated According to Element Sets

\begin{tabular}{lccc}
\hline \multicolumn{1}{c}{ Element Set } & P & NP & Other \\
\hline Line Segments & 7 & 8 & 1 \\
Gray Disks (IR = 3.5) & 8 & 7 & 1 \\
Gray Disks (IR = 2) & 5 & 9 & 2 \\
Gray Disks (IR = 1.25) & 7 & 8 & 1 \\
Colored Disks & 6 & 8 & 2 \\
\hline
\end{tabular}

Note-IR = intensity ratio.

A breakdown of frequencies of interpretations by element sets is presented in Table 1. The irrelevance of identity preservation appears to recur within every element set. Testing this point statistically is problematic, since every subject contributed four observations to this table. However, to get a feeling, a chi-square value was computed nevertheless $\left[\chi^{2}(4)=.95, p>.9\right]$.

The "cha-cha-cha" episode. A report was classified as circular if the motion reported was continually clockwise, was continually counterclockwise, or switched from one direction to another but not more than three times. Any such circular motion violates the preservation of identity. Two subjects who were presented arrays of line segments reported that the segments changed orientation but did not change location. This interpretation was also considered as violating preservation of identity. The only interpretation that does preserve the identity of elements is a 30-deg back-and-forth tilting motion. For the line segments, four subjects did not preserve identity and none did. For the gray disks, one preserved identity, nine did not, and the reports of another two were unclear. For the colored disks, however, all four subjects did preserve identity and none did not. Thus, there appears to be a clear difference between the motion impression created by color disks arrays and the other types of array. To test it, the two other types were collapsed in one category and unclear interpretations were eliminated. The association between preservation of identity and type of elements was found significant by a two-tailed Fisher exact probability test $(\mathrm{p}<.01)$.

Thus, while the "seesaw" episodes indicated no noticeable effect of any aspect of element identity on the type of motion experienced, the "cha-cha-cha" episode suggested that such an effect might nevertheless exist. But what is the critical difference between the "seesaw" episodes on the one hand and the "cha-chacha" episodes on the other hand that led to the discrepant findings with regard to the effect of color disparity? One major difference is the interelement distance. To get an idea of whether this hypothesis could be supported, I had several people, including myself, watch the "seesaw" episodes from a distance about three times as large. Their experience was completely unchanged. An alternative explanation may be that the critical variable is the ratio between the interelement distance and the element size. Thus, I reduced this ratio in the "seesaw" 
episodes by a factor of four and presented the new versions to different subjects.

\section{EXPERIMENT 2}

\section{Method}

Experiment 2 differs from Experiment 1 only with regard to stimuli and the design. Only the four "seesaw" episodes were administered, and they differed from the corresponding episodes in Experiment 1 in only one aspect: The distance between element centers in each array subtended about $0 \mathrm{deg} 47 \mathrm{~min}$. Two element sets were used, line segments and colored disks. Each of the four episodes appeared equally with each element set across subjects. Each subject saw one of the "loaded seesaw" episodes with line segments as elements and the other episode with colored disks; the same was true for the other two episodes. The order of episodes was determined by a 4 by 4 Latin square. Each subject was assigned to one sequence of the Latin square. The standard exposure was $7 \mathrm{sec}$, and the subject was given another $2 \mathrm{sec}$ for verification of report in case he was not sure about it. Sixteen nonpaid undergraduates at the University of Haifa participated in the experiment. All subjects had normal or corrected-to-normal vision.

\section{Results}

In general, in this experiment, subjects appeared to be less able to describe their experience and less certain about the description. This was especially pronounced with line segments when they appeared to change orientation. Change of element identity was then inferred from the direct report that the segments had changed orientation.

The results of this experiment were generally congruent with the results of Experiment 1. When data from the "horizontal seesaw" and "vertical seesaw" episodes were pooled across element sets, no association between the observed interpretation of an episode and the one predicted by identity preservation was found [Cochran's $\mathrm{Q}(1)=2.0, \mathrm{p}>.10]$. Identity was preserved in both of those episodes for 2 subjects, whereas 14 subjects reported identical interpretations in both episodes (binomial probability $=.004$ ). The proportions of identity-preserving interpretation within the two element sets were identical ( 9 of 16).

For the "loaded seesaw" episodes, again no association was found between the interpretation and the type of episode [Cochran's $\mathrm{Q}(1)=.33, \mathrm{p}>.50$, after eliminating three subjects whose data were unclear]. No subject preserved the identity in the first interpretation in both episodes, yet two subjects switched to the identitypreserving interpretation. For two more subjects, identity was not preserved in both episodes, and nine subjects reported identical interpretations in both episodes. Thus, the number of subjects with identical first interpretations was 11 (binomial probability $=.02$ ). For line segments, there were 8 identity-preserving interpretations out of 13 , and for colored disks, there were 3 such interpretations out of 12 (one interpretation was unclear). The difference between these proportions was nonsignificant [Cochran's $\mathrm{Q}(1)=2.27, \mathrm{p}>.10]$.
Thus, the results do not lend any support to the hypothesis that the previous failures of element identity to affect apparent motion in the "seesaw" episodes are due to the large distance/size ratio.

One might feel uncomfortable about the use of naive subjects, each contributing a single observation per episode. Hence, I conducted a further study that addresses these points.

\section{EXPERIMENT 3}

\section{Method}

Nine episodes were presented. The "cha-cha-cha" episode was presented once, and each of the "seesaw" episodes was presented in the two versions used for the two previous experiments. All nine episodes were used with Element Set 5 (namely, the hue set). Each subject was presented a sequence of 180 presentations, 20 for each episode, randomly intermixed. Each presentation lasted 5 sec. Subjects were asked to assign the perceived motion in the "seesaw" episodes to one of four categories (vertical, horizontal, positive diagonal, negative diagonal) and the motion in the "cha-cha-cha" episode to one of three categories (tilt, clockwise, and counterclockwise). Subjects were four research assistants and myself.

\section{Results}

For each pair of the "seesaw" episodes and each of the subjects, the association between the episode and the subject's response was tested by means of a chi-square test. The results for each subject for the large unloaded seesaw, large loaded seesaw, small unloaded seesaw, and small loaded seesaw, respectively, were: Subject R.B., $.00, .12, .00, .50$; Subject R.K., $.00, .00, .00, .00$; Subject D.N., .00, .46, .00, .43; Subject M.S., .02, .00, .47, .29; and Subject N.A., 26.26, 32.48, 27.92, 35.10. Only the last four were significant. Thus, for four of five subjects, there was no indication of any tendency to preserve hue in the motion perceived.

In the "cha-cha-cha" episode, four subjects selected most often the hue-preserving tilt interpretation (binomial probabilities of $.41, .03, .00$, and .02 for Subjects R.K., D.N., M.S., and N.A., respectively). The fifth subject, R.B., selected a counterclockwise motion in all trials.

Thus, as in the experiments with naive subjects, there was a contrast between the irrelevance of hue for the interpretation of motion in the "seesaw" episode and the indications for some tendency to preserve hue in the "cha-cha-cha" episode.

\section{DISCUSSION}

The discrepancy between the effects of preservation of identity in the two types of episode suggests that the factors operating against the identity-preserving motion were different in each of them. One could think of two factors. The first factor is a tendency for continual motion. Conceivably, the 12 locations occupied by elements in the "cha-cha-cha" episode suggest the pattern of a regular dodecagon more than the 4 element locations in the "seesaw" episodes suggest the pattern of a square. That may explain why the frequency of circumferential motion in the "cha-cha-cha" episode was much higher than in the 
"seesaw" episodes. A second factor that may be termed axis preference participates in determining which particular back-andforth motion wiil be perceived. In the "seesaw" episodes, there appeared to exist for almost every subject a strong preference of the vertical axis over the horizontal one (or vice versa) and of the negative diagonal over the positive one (or vice versa). However, this factor could not be very influential in the "cha-cha-cha" episode, because every back-and-forth interpretation involved motion along three different axes.

The axis-preference factor that operated in the "seesaw" episodes was presumably stronger than the tendency for continual motion that counteracted preservation of identity in the "cha-cha-cha" episode. Preservation of hue was more potent than preservation of brightness or orientation and was strong enough to override the tendency for continual motion but was outweighed by the factor of axis preference.

It may be concluded that motion analysis is neither totally dependent on nor completely independent of analyses of shape, hue, brightness, and so on. Those analyses may be parallel and interdependent. The final output of one type of analysis may be affected by whatever intermediary output it receives from the other one. Thus, the percept depends on the relative rate of analysis. When static analysis is simple, its output may arrive early enough to participate in the formation of motion. For example, noticing considerable hue or size disparities may be sufficiently fast to have a potential bias on motion perception, whereas the rate of figural analysis may be typically too slow to enable any impact of figural identity on the motion perceived.

\section{REFERENCES}

Burt, P., \& Sperling, G. Time, distance, and feature tradeoffs in visual apparent motion. Psychological Review, 1981, 88, 191-195.

Higarnson, G. D. Apparent visual movement and the Gestalt. Journal of Experimental Psychology, 1926, 9, 228-252.

Kolers, P. A., \& Pomerantz, J. R. Figural change in apparent motion. Journal of Experimental Psychology, 1971, 87, 99-108.

Navon, D. Irrelevance of figural identity for resolving ambiguities in apparent motion. Journal of Experimental Psychology: Human Perception and Performance, 1976, 2, 130-138.

NEFF, W. A. A critical investigation of the visual apprehension of movement. American Journal of Psychology, 1936, 48, 1-42.

Neuhaus, W. Experimentelle untersuchung dir scheinbewegung. Archiv für die Gesamte Psychologie, 1930, 75, 315-458.

Orlansky, $J$. The effect of similarity and difference in form on apparent visual movement. Archives of Psychology, 1940, 246, 85.

RATTLETr, A. A study of visual movements determined by form, colour or brightness. Acta Psychologica, 1956, 12, 65-75.

(Received for publication December 7, 1982.) 